\title{
Diet and physical activity behaviour in nurses: a qualitative study
}

\author{
Luciana Torquati ${ }^{\mathrm{a} *}$, Tracy Kolbe-Alexander $^{\mathrm{b}}$, Toby Pavey ${ }^{\mathrm{b}}$, Christina Persson $^{\mathrm{c}}$ and Michael Leveritt ${ }^{\mathrm{a}}$ \\ ${ }^{a}$ Centre for Dietetics Research, School of Human Movement and Nutrition Sciences, The University of \\ Queensland, Cnr Blair Drive \& Union Road, St Lucia Campus, Brisbane, QLD 4072, Australia; \\ ${ }^{b}$ Centre for Research in Exercise, Physical Activity and Health, School of Human Movement and \\ Nutrition Sciences, The University of Queensland, Cnr Blair Drive \& Union Road, St Lucia Campus, \\ Brisbane, QLD 4072, Australia; \\ ${ }^{c}$ Department of Molecular and Clinical Medicine, Sahlgrenska Academy, University of Gothemburg, \\ Medicinaregatan 3a-5b, Göteborg 40530, Sweden
}

Issue addressed. Previous research has shown that approximately $60 \%$ of nurses in Australia are overweight or obese, insufficiently active and have an unhealthy diet. The aim of this study was to gain an understanding of nurses' determinants contributing to these behaviours. This will inform a needs assessment for a future Workplace Health Promotion Program (WHPP) in this group.

Methods. Four focus group discussions $(n=17)$ were conducted with a convenience sample of nurses aged 25-59 years from three hospitals in the Brisbane metropolitan area. Questions addressed barriers and motivation towards diet and physical activity (PA), and suggestions for future WHPP. Transcripts were analysed with Nvivo10 following a thematic analysis with a realistic approach, using Self-Determination Theory as a framework.

Results. Work environment was the main barrier for healthy diet behaviours. Long working hours and lack of breaks challenged nurses' self-control and self-regulation when making dietary choices. Fatigue was the main barrier for PA. However, relaxation, feeling energized before work, and better sleep after working night shifts motivated nurses to do PA. Social environment at work seemed to be an effective external motivation to encourage healthy diet and regular PA. Goal-setting, selfmonitoring and social support at work were identified as potential WHHP strategies.

Conclusion. The workplace and job demands negatively impacts nurses' lifestyle behaviours. Future interventions should include social support from colleagues, which could motivate nurses to make healthier food choices at work and be more active outside work.

Keywords: Nutrition, Physical Activity, Qualitative research, Workplace Health Promotion

\section{Word count: $4000 \mathrm{w}$}




\section{Introduction}

Physical activity (PA) and poor diet have been identified as two of the main risk factors for non-communicable diseases, with these behaviours accounting for $10 \%$ of global years lost to ill-health, disability or early death (Lim et al. 2012). Promoting diet and PA remains a challenge due to the complex interplay between individuals' behavioural patterns and their environmental determinants (Biddle and Mutrie 2007, Wendel-Vos et al. 2007, Stafford et al. 2007, Gortmaker et al. 2011). Occupational demands, like long working hours and job requirements, also play an important role on individuals' lifestyle (Quintiliani, Sattelmair, and Sorensen 2007). Nursing, for example, is a profession shown to contribute to poor diet and PA behaviours, because of the stressful job demands, long working hours, and night or rotating shifts (Wong et al. 2010, Huntington et al. 2011, Schluter et al. 2011). Nurses' diet often comprises of irregular mealtimes with high-fat and high-sugar meals, and frequent snacking of energy dense foods (Faugier et al. 2001, Cheung 2003, Zapka et al. 2009, Wong et al. 2010). Nurses' PA behaviour is characterised by $50 \%$ not meeting recommended PA guidelines (Zapka et al. 2009, Blake et al. 2011, Sumaira, Holly, and Mark 2011).

Despite the need to improve nurses' diet and PA behaviours (WHO 2010, Verweij et al. 2011), few lifestyle interventions have been implemented and reported in the literature (Chan and Perry 2012, Torquati et al. 2015). Our recent systematic review found only seven diet and PA Workplace Health Promotion Programs (WHPP) in this population (Torquati et al. 2015). Some strategies used in these WHPP included pedometers, walking stations, nurse champions and information sessions. However, because of the low number and quality of studies, authors could not conclude whether these interventions were effective in changing nurses' behaviour and improving their health. Therefore, better-designed and effective WHPP targeting nurses are needed. 
For effective WHPPs, the study design is guided by the target group's determinants of both current behaviours and desired outcomes (Brug, Oenema, and Ferreira 2005). A rationale linking the choice of intervention methods (strategies and theoretical constructs) to the target group's determinants of behavior is then developed. A needs assessment explores such determinants through qualitative research (e.g. surveys, focus groups) and literature review (Green, Kreuter, and Green 2005, Bartholomew et al. 2011). Few studies have conducted qualitative research on diet and PA behaviours in the nursing population, which are limited to the impact of night shift or to the barriers of diet and PA behaviour (Novak and Auvil-Novak 1996, Person and Mårtensson 2006).

With the use of an appropriate theoretical framework, qualitative data identifying both facilitators and barriers to diet and PA behaviours, could be used as part of a needs assessment in this group. Nurses' opinions on preferred intervention strategies should also be included, as incorporating suggestions from the target group has been shown to increase the effectiveness, flexibility and sustainability of future interventions (Bartholomew et al. 2011). Flexible and sustainable WHPP are particularly important in this hard-to-reach group, therefore it is important to identify factors that could motivate nurses to improve their diet and PA behaviours.).

Self-Determination Theory (SDT), defines the intention to perform a behaviour (motivation) as a condition and key factor of behaviour's implementation (Ryan et al., 2008). Motivation could be intrinsic or extrinsic depending whether it is coming internally from the individual (i.e. behavior is personally important and interesting), or externally from the environment (i.e. social rules, peer pressure) Thus, SDT is useful for understanding motivational processes and adherence to health behaviours ( $\mathrm{Ng}$ et al. 2012). Understanding 
the interplay between determinants at a personal, social and environmental level in the target group is a necessary step to effectively change diet and PA behaviours (Brug, Oenema, and Ferreira 2005).

This study aims to explore perceived barriers and enablers of healthy diet and PA behaviours, in a group of Australian nurses. The data obtained will be used as part of a needs assessment to inform a future WHPP aimed to promote healthy diet and PA in nurses.

\section{Methods}

Focus group (FG) interviews were used to gather qualitative data. The study aimed to have four to ten participants per FG, with group allocation based on homogeneity (Doody, Slevin, and Taggart 2013a). To promote a comfortable and casual environment, and encourage attendance, the FGs had the format of morning or afternoon tea group discussion sessions.

\section{Setting}

This study was conducted in three hospitals from the metropolitan area of Brisbane (QLD). Nursing Unit Managers (NUMs) based at hospitals identified as potential sites for future WHPP were invited to participate in the research. The NUMs were asked to distribute information related to this study to their staff.

\section{Participants}

After NUMs sent all of their nurses an email informing them of the study, posters were placed in common staff areas (i.e. staff rooms, canteens, notice boards). Nurses interested in 
participating were instructed to contact the FG moderator (LT) to set a date and time to attend the FGs. They comprised a convenience sample that took part in this study.The inclusion criteria for participation were: (1) currently working as a nurse (e.g. registered nurse, clinical nurse) and (2) employed at one of the potential intervention sites for a future intervention.

\section{Procedure}

Before the focus group started, information sheets about the study were given to participants. After participants finished reading, and eventual questions answered, participants were asked to sign a consent form and provide demographic information.. The facilitator reminded participants that the FG will be audio-recorded, and that they were free to leave at anytime. The only incentives that were provided were beverages and light snacks. This study protocol was approved by the Mater Health Services HREC (Human Research Ethics Committee) and The University of Queensland MREC (Medical Research Ethics Committee).

FGs $(n=4)$ took place in a private room at each hospital, at a time that was convenient for participants, which was either just before or after their shifts. Nurses from similar units, shifts and age range were grouped to create homogenous groups. Homogeneity is important, as participants are more willing to share personal experiences when they feel similar to others in the group (Doody, Slevin, and Taggart 2013b). FGs were organised and held until reaching saturation point, when no new information was found (Doody, Slevin, and Taggart 2013a). For this reason, we did not predetermine a sample size. The moderator (LT) facilitated the discussion, and an assistant (CP) observed and took notes (in addition to the audio recordings) about speakers' name and body language to facilitate transcription and interpretation of quotes.

\section{Interview guide}


An interview guide was constructed comprising of 10 short open-ended questions formulated to sound conversational (see Table 1) and to facilitate discussion of personal opinions and experiences about health, nutrition and PA (Krueger and Casey 2002). Questions related to the participant's interests and preferences for a future WHPP were also included.

The questions in the interview guide were selected to fit study's aim and designed to be well phrased, sequenced in a logical order, and easy to understand for the participants (Krueger and Casey 2002). We follow Krueger and Casey (2002) approach and Ethics Committee feedback when developing and scheduling questions. Questions were lined-up from general to more specific, to foster conversation among the group and facilitate group dynamics. After the first FG, some questions were modified slightly to generate better interaction and discussion between participants. For example, 'Do you consider yourself active?' was changed to 'What motivates you to engage in physical activity?'.

[Table 1 here]

\section{Data analysis}

The audio-recordings were transcribed verbatim and merged with notes taken during the interviews. The transcription was imported and coded in Nvivo10.0. Data was sorted and organized following the framework described by Krueger and Casey (2002). Data analysis followed thematic analysis with a realistic approach, where information was collated in themes. Themes aimed to report relevant information to the research question and to represent a patterned response through the data set (Braun and Clarke 2006). By using this technique, the researcher first grouped statements into units using different coding. Similar coding units were grouped into different categories (nodes). Finally, the nodes were clustered into general themes that summarised the node content. Quotes were chosen from the transcript to represent 
each category in the results section. Two researchers (LT, CP) worked independently with the coding and categorisation process, and when the analysis was finished, results were compared. The analysis was separately reviewed by the remaining authors (ML, TP, TKA). Deciding how well eventual themes would fit the focus of the study solved eventual disagreements in themes and codes allocation. However, no substantially different interpretations emerged. Finally, a summary including themes and categories was sent to all participants to check whether the analysis has reflected the participants' views accurately.

We used the Self-Determination Theory (SDT) as a framework for interpretation of themes. SDT states that humans have an inborn motivation to self-regulate health behaviors (Ryan and Deci 2000). This theory is considered useful for understanding motivational processes and adherence to health behaviours ( $\mathrm{Ng}$ et al. 2012). Motivation is seen as a continuum from intrinsic to extrinsic motivation, and varies in the extent to which it is autonomous (self-determined) or controlled. This and other social theories explain how individual intentions and choices are mediated by social influences and environmental factors in PA and diet behaviours (Brug, Oenema, and Ferreira 2005). Results were interpreted considering SDT motivation and barriers to self-determined diet and PA behaviours, and are shown in Figure 1.

\section{Results}

Four FGs were conducted with a total of $n=17$ nurses, who were mostly female and registered nurses. The average age was 40 years old with mean working experience of 15 years (see Table 2). The FGs ranged from 25 to 70 minutes, and the average duration was $48 \mathrm{~min}$.

Quotes were interpreted and classified in three main themes in line with the research questions: 1) Workplace determinants for diet and PA; 2) Personal determinants for diet and 
PA; 3) Strategies for futures WHPPs. Finally, the resulting determinants of diet and PA were organised in a framework based on Self-Determination Theory (Ryan and Deci 2000) (see Fig. 1).

\section{[Table 2 here]}

\section{Workplace determinants for Diet and PA}

\subsection{Night shift}

Working the night shift was reported as one of the major extrinsic barriers for both following healthy diet and participating in PA. Some of the situations influencing diet behaviour included having irregular meal patterns and lack of breaks at work, which were more common during night shifts, leading to poor food choices at mealtimes. Nurses reported that they continuously consumed high-energy snacks, like chocolate and crisps, to help them stay awake during their night shift.

'You eat crisps to stay awake all night and coke....and by the end of the night you feel revolting!' (N2 (Nurse 2), FG2 (Focus Group 2))

'You got your breakfast and go to bed, then you wake up at lunchtime starving and eat then. And then you eat again all through the night.' (N2, FG3)

\subsection{Long working hours and breaks}

Long shifts ( $>8 \mathrm{~h})$ and insufficient breaks were described as extrinsic barriers to healthy diet. The work demands during a shift sometimes made it challenging to find opportunities to take a break. This led to nurses eating unhealthy food and overeating in general, which was a consistent topic raised in all the FGs. Nurses explained that they would eat as much as they can when given the opportunity to take a break during long shifts. In some cases, they would even eat irrespective of being hungry or not. 
'In some particular days you just stuff it down at the end (end of shift)' (N4, FG1)

'You don't know when you will be able to eat again' (N2, FG4)

\subsection{Food availability}

The nurses reported that the quality of their food choice was influenced by the food available in the hospital wards. For example, consistent presence of chocolates and treats in the wards would make nurses continuously snack on these, because they are available and accessible. As a result, this extrinsic factor challenged nurses' intrinsic motivation of self-control when making dietary choices. Participants perceived this as one of the main causes of overweight in this population.

'You can't avoid the eating during the shift, because the nursing station is full of chocolate' (N1, FG2)

'I'm not normally a chocolate person ...except if it's right there in from of you' (N4, FG4).

'It's all very nice, but you can see yourself expanding over time' (N2, FG4).

On the other hand, nurses explained how having healthier options available at work could help them make better food choices. They reported that they would make healthy food choices when the healthy options are available to them.

'It would help with diet if there would be food provided... healthy food' (N1, FG3)

\subsection{Work facilities to enable healthy behaviours}

Some nurses mentioned their willingness to integrate PA with work when possible, by actively commuting to work or doing exercise before shifts. However, the lack of adequate 
shower facilities at work was an extrinsic barrier. Participants reported that small and insufficient shower facilities discouraged some nurses from actively commuting to work. Another barrier was the cost associated with using the onsite gym, which was small and accessible for both staff and patients, thus not "staff only".

'You get a reduced staff rate ... and that's a big barrier. That's a big barrier when for most people I think their budget is pretty tight.' (N3 \& N1, FG2)

\section{Personal determinants for Diet and PA}

Participants described their perceived benefits and enjoyment of regular PA. These benefits included reducing job stress and fatigue, feeling energised before work and relaxed after work. However, they were not always able to prioritise PA because other commitments, such as social and family activities, were considered more important.

\subsection{Fatigue}

Both working long hours and being tired were the main factors influencing PA. Most of the nurses mentioned lack of time or having competing priorities, such as family or social commitments. Shift work was an extrinsic barrier towards nurses' ability to keep active, mostly because of the associated fatigue. Even those nurses who were regularly physically active mentioned these barriers.

'I just find that I'm too tired' (N1, FG4)

'I want to do exercise, and I find I can't do that with the shift work' (N3, FG2)

'On a 12-h shift, I could not cycle in and cycle home' (N2, FG2) 


\section{$2.2 \quad$ Job stress}

The participants directly linked job stress to dietary habits. Stress was related to emotional eating and craving on high-fat high-sugar food, such as chocolate, candy and fast food. When they tried to be mindful about their diet, being stressed or fatigued were enough to undermine this behavior leading to lack of self-control over diet.

'You have a bad day, and before you know it you have five (chocolates) in your mouth' (N4, FG3).

'Your ability to be disciplined changes... because you are so tired' (N2, FG1).

'If you are depressed or something is upsetting you, you make comfort eating' (N1 \& N2, FG1).

\subsection{Enjoyment and stress relief}

Nurses were aware of the positive impact of PA on their health and well-being. They viewed PA as a way to relieve stress and de-attach from work, representing intrinsic motivation for nurses. The main strategy to fit PA into their busy schedule was to exercise either before or after work, which was associated with improved health and increased energy levels at work. Two nurses in the total group reported that they enjoyed cycling to work and this was associated with improved quality of sleep, especially after working night shift.

'I just go for a walk or something and that's really really helpful. It de-stresses you' (N4,

FG4)

'I find that if I am doing a little bit of physical activity before work, I feel energised.'

(N1, FG1)

'I'd ride home and I'd definitely sleep better yeah' (N2, FG2)

\section{Strategies for future WHPPs}


Nurses felt their knowledge on healthy lifestyle, specifically diet and PA, were not enough to overcome the barriers towards improving these behaviours. They expressed an interest in WHPP to help them improve their lifestyle, and to help them overcome the barriers they experienced. Considering the determinants previously discussed and participants' suggestions, the nurses emphasized that one of the key concepts for future interventions is to keep them 'simple'.

'Because I know... what's healthy and what's not. I can't stop it putting food into my mouth!! You know?...I know the types of exercise I could be doing to reduce it all, and knowing that doesn't help and hasn't help, clearly.' (N2, FG2)

\subsection{Social support}

Social-support for diet and PA appeared to be a strong extrinsic motivator for nurses. For example, nurses suggested they could limit the intake of unhealthy snacks during night shift if there was a group/ward commitment towards this goal. In terms of PA, nurses indicated that social support could promote PA engagement. Exercising with colleagues was described as a way to socialise outside work, have fun, and promote team-building.

'We said don't bring any chips, don't bring any cake, we are just gonna bring sensible food' (N3, FG2)

'It was more about that collective group exercising together apart from doing what our normal job was. And it was really good' (N2, FG4)

\subsection{Social media and apps}

Participants discussed the use of technology to promote social support, communication and motivation. Some reported they tried to use social media (Facebook) to improve their diet and 
PA in the past. Apps were perceived as a strategy that could help them stay motivated and allow the nurses to receive weekly reminders.

'I suppose those app like say something positive everyday (...) or something like that, sort of encouraging the group' (N1, FG1).

'We had this kind of Facebook thing like 10-week challenge ...there were a couple of us doing it too' (N2, FG4).

\section{$3.3 \quad$ Self-monitoring}

Self-monitoring was suggested as a simple way to improve current diet and PA behaviours. Having a pedometer to count daily steps accumulated was one of the strategies suggested to promote PA at work and at home. It could lead to intrinsic motivation to move more and could be used for a workplace challenge.

'A pedometer is a good motivational tool. Could you provide pedometers for everybody maybe? Like a workplace step-challenge or something...it could work!' (N2, FG4)

\subsection{Goal-setting}

Setting goals to promote healthy diet and increase habitual levels of PA was identified as a feasible and acceptable strategy. Goals could be set using a mobile app and these could promote intrinsic motivation towards diet and PA behaviours. Goal-setting was associated with social support, for example, having team-based challenges and goals.

'I think you have to have an app which has set 5-6 random goals... and then maybe have a different section where you kind of can put your own goals' (N1, FG2).

'I heard you gotta write goals, and you gotta stick to them and reward yourself kind of stuff' (N4, FG3) 


\subsection{Workplace culture}

Participants often reported that incorporating PA in the workplace would help them to be more active. This was particularly important for those who found it difficult to exercise before or after work. A change in the workplace culture was seen as a necessary step to enable healthier lifestyle practices at work. This could represent an important extrinsic motivation towards healthy eating at work. Importantly, participants felt this change should come from management.

'And sort of a culture thing, where there is an encouragement of healthy lifestyle practices, encouraging commitment within departments to do that kind of thing'

(N3, FG2)

'Making it a policy of the hospital... Get up to management and go "this is what I want to do, bring this in and make everyone do it".' (N2, FG3)

\section{Interplay of behaviour determinants and Self-Determination Theory}

The resulting determinants for diet and PA were organised in a framework based on the SelfDetermination Theory (see Figure 1). This diagram shows the interplay of intrinsic (individual) and extrinsic (social and work environment) motivation and barriers on diet and PA. For example, health, wellbeing and weight management were intrinsic motivation cues for healthy eating and being active. However, fatigue and lack of self-control were intrinsic barriers to healthy behaviour, with work colleagues representing both extrinsic motivation and a barrier to healthy diet and PA.

[Figure 1 here] 


\section{Discussion}

The focus of this study was to explore perceptions and determinants of healthy diet and PA behaviors in nurses. This represented a component of a needs assessment to inform future WHPP strategies. Using the Self-Determination Theory as a framework, we identified the following factors influencing nurses' diet and PA behavior: individual barriers and motivation (intrinsic), social support (extrinsic) and work environment (extrinsic). One of the main findings was that extrinsic factors were perceived to have a greater influence on dietary behaviour than intrinsic factors.

The major factor influencing diet was a complex cycle between lack of breaks at work, being hungry, decreased self-control and leading to overeating and/or making unhealthy choices. This provided an insight of why long shifts and night shifts have been previously portrayed as a barrier (Novak and Auvil-Novak 1996, Person and Mårtensson 2006). In particular, nurses working night shift have been described as more likely to adopt abnormal eating behaviors, like consumption of high-fat foods and snacks (Wong et al. 2010). As with a previous study, our participants associated night shift work with increased body mass over time (Zhao et al. 2011). These results suggest the need to develop strategies for nurses to better cope with the negative impact of night shift to limit the onset and further development of obesity.

Job demands and work environment were extrinsic factors negatively impacting diet and PA. An example of this was availability of chocolates and candy at work and nurses eating them just because "they were there". Cheung (2003) also found a high consumption in nursing staff with an average of 5.4 chocolate portions/day being eaten. Job demands and consequent fatigue discouraged nurses to be active after work and during their days off. Fatigue, long working hours and lack of time were found to be main barriers to PA, which is 
congruent with previous studies (Novak and Auvil-Novak 1996, Person and Mårtensson 2006, Zapka et al. 2009, Nahm et al. 2012). Participants reported that having the necessary knowledge of healthy behaviours was insufficient to counteract these barriers. They discussed the need for a WHPP that could help them cope with their barriers and encourage them to improve their diet and PA behaviours.

Based on SDT, the mismatch between knowledge and behavior could be explained as decreased motivation and self-efficacy to perform healthy behaviours, because of low perceived competence and autonomy (Williams et al. 2002, Teixeira et al. 2012). Low perceived competence is when individuals are not motivated enough to prioritise a behavior when faced with other interests or demands of their time (i.e. participants not exercising because they prefer to doing other things in their free time). Decreased autonomy is when an individual starts and fails to maintain a behavior (i.e. being mindful about food) by feelings of "having to" (controlled motivation) rather than volition, importance or enjoyment of that behavior (autonomy). Nurses' difficulty to engage and maintain healthy behaviours further highlighted the need for a WHPP with appropriate strategies to improve their autonomy and competence.

One aspect that could contribute towards increasing nurses' autonomy and competence in PA, for example, is experiencing and focusing on the benefits of PA. Participants in the study felt motivated to engaged in PA because of its health benefits. They described these as experiencing increased levels of energy before work, improved sleep quality especially after working night shift, and decreased stress levels. Further, nurses have previously described PA as a stress coping mechanism (Person and Mårtensson 2006). Given the stressful nature of nurses' job, the positive effects of PA, and the high incidence of overweight and obesity in this group, PA should be promoted in future WHHP both as a stress coping and weight management strategy. 
The social and work environments were identified as the main factors that should inform the design and components of future intervention program. Peers in similar situations, who provide support, understanding, and coping strategies, stimulate social support (Munson et al. 2013). A recent intervention study showed that PA increased more in a walking support group and pedometers compared to the pedometer only group (10,064 vs 12,472 steps/day; $\mathrm{p}<0.05)$ (Bravata et al. 2007). Support from colleagues for the promotion and commitment of limiting the amount of unhealthy food at work was mentioned across all groups. Social support from work colleagues could motivate nurses to make healthier food choices at work and be more active outside work.

Other strategies for future WHPP could include goal-setting and self-monitoring, as suggested by the participants in this study. Mobile apps could assist promoting these approaches. Diet and PA self-monitoring has been shown to significantly reduce energy intake $(1437 \pm 188$ vs $2049 \pm 175 \mathrm{kcal} /$ day, $\mathrm{p}=0.01)$ and BMI $(31.5 \pm 0.5$ vs $32.5 \pm 0.5 \mathrm{~kg} / \mathrm{m} 2$; $\mathrm{p}=0.02$ ) in app users compared to non-users at 6 months (Turner-McGrievy et al. 2013). Participants in our study also referred to pedometers as a simple strategy to monitor their PA. Pedometers are widely used in health promotion, and have been shown to successfully increase PA (Bravata et al. 2007). Pedometers and apps have the potential to be included in future WHHP for nurses, as they are a practical low-cost tool to promote and motivate selfmonitoring and goal-setting (Bassett Jr and John 2010, Al Ayubi et al. 2014).

Participants felt that healthier behaviours could be promoted at their workplace if management were involved. (Lemon et al. 2010) showed how hospital employees' job perceptions changed with a diet and PA WHPP. Although BMI changes were small, employees' perceptions of organisational commitment significantly improved at 12 and 24 months. Therefore, having management involved in future WHPP will not only encourage 
enrolment and facilitate participation, but will also highlight the importance of the program itself.

One of the strengths of this study was the use of focus groups as a method to explore nurses' perceptions of PA and diet. Having semi-structured questions generated discussion among participants, providing richer data than previous similar studies (Novak and AuvilNovak 1996, Person and Mårtensson 2006, Zapka et al. 2009, Nahm et al. 2012). Although some barriers towards diet and PA are well known in the literature, by using SDT as a framework, we were able to shed light on nurses' motivation to adopt healthier behaviours. As SDT states, motivation is the key for behavior change and maintenance of the new behaviours (Ryan et al. 2008). Another strength was having a question guide checked and adapted to avoid irrelevant or ambiguous questions. Having a question guide ensured consistency across groups and facilitated the comparison of data between groups.

One limitation was the inclusion of one part-time nurse and four clinical nurses and nurse educators. These nurses may have not been exposed to the job environment as much as the other participants. In particular, clinical nurses and nurse educators have a more officebased job with limited shifts on the floor. To overcome this limitation, these participants were asked to answer questions referring to their experience when working on the floor or when they used to be full-time nurses. Given the lack of time of most nurses, recruitment was the main challenge. The majority of the contacted nurses was busy and had little time to participate. As a result, it was difficult to find participants willing to stay outside working hours and to find a suitable day and time for the each FG. For this reason, one of the FGs lasted less than one hour.

\section{Conclusion}


Work environment was the major barrier towards healthy diet, while fatigue after work challenged physical activity behaviour. Long working hours and lack of breaks challenged nurses' diet self-control and self-regulation. Social environment at work seemed to be an effective external motivation to diet and exercise. In the nursing population, future WHPP should foster social support from colleagues and implement workplace environmental changes to facilitate healthier behaviours. 


\section{References}

Al Ayubi, S. U., B. Parmanto, R. Branch, et al. 2014. "A Persuasive and Social mHealth Application for Physical Activity: A Usability and Feasibility Study." JMIR mHealth and uHealth 2 (2):e25.

Bartholomew, L. K., G. S. Parcel, G. Kok, et al. 2011. Planning health promotion programs: an intervention mapping approach. San Francisco: Jossey-Bass.

Bassett Jr, D. R., and D. John. 2010. "Use of pedometers and accelerometers in clinical populations: validity and reliability issues." Physical therapy reviews 15 (3):135-142.

Biddle, S. J., and N. Mutrie. 2007. Psychology of physical activity: Determinants, well-being and interventions: Routledge.

Blake, H., S. Malik, P. K. H. Mo, et al. 2011. "'Do as I say, but not as I do': Are next generation nurses role models for health?" Perspect Public Health 131 (5):231-239. doi: Doi 10.1177/1757913911402547.

Braun, V., and V. Clarke. 2006. "Using thematic analysis in psychology." Qualitative research in psychology 3 (2):77-101.

Bravata, D. M., C. Smith-Spangler, V. Sundaram, et al. 2007. "Using pedometers to increase physical activity and improve health: a systematic review." JAMA 298 (19):2296-304. doi: 10.1001/jama.298.19.2296.

Brug, J., A. Oenema, and I. Ferreira. 2005. "Theory, evidence and Intervention Mapping to improve behavior nutrition and physical activity interventions." International Journal of Behavioral Nutrition and Physical Activity 2 (1):2.

Chan, C. W., and L. Perry. 2012. "Lifestyle health promotion interventions for the nursing workforce: a systematic review." $J$ Clin Nurs 21 (15-16):2247-61. doi: 10.1111/j.1365-2702.2012.04213.x.

Cheung, S. T. 2003. "The effects of chocolates given by patients on the well-being of nurses and their support staff." Nutr Health 17 (1):65-9.

Doody, O., E. Slevin, and L. Taggart. 2013a. "Focus group interviews in nursing research: part 1." Br J Nurs 22 (1):16-9.

Doody, O., E. Slevin, and L. Taggart. 2013b. "Focus group interviews part 3: analysis." British journal of nursing (Mark Allen Publishing) 22 (5):266.

Faugier, J., J. Lancaster, D. Pickles, et al. 2001. " Barriers to healthy eating in the nursing profession: Part 1." Nursing Standard 15 (36):33-36. 
Gortmaker, S. L., B. A. Swinburn, D. Levy, et al. 2011. "Changing the future of obesity: science, policy, and action." The Lancet 378 (9793):838-847. doi: http://dx.doi.org/10.1016/S0140-6736(11)60815-5.

Green, L. W., M. W. Kreuter, and L. W. Green. 2005. Health program planning: an educational and ecological approach. 4th ed. Boston: McGraw-Hill.

Huntington, A., J. Gilmour, A. Tuckett, et al. 2011. "Is anybody listening? A qualitative study of nurses' reflections on practice." $J$ Clin Nurs 20 (9-10):1413-22. doi: 10.1111/j.1365-2702.2010.03602.x.

Krueger, R. A., and M. A. Casey. 2002. "Designing and conducting focus group interviews." Social Analysis, Selected Tools and Techniques”, Krueger, RA, MA Casey, J. Donner, S. Kirsch and JN Maack:4-23.

Lemon, S. C., J. Zapka, W. Li, et al. 2010. "Step ahead a worksite obesity prevention trial among hospital employees." Am J Prev Med 38 (1):27-38. doi: 10.1016/j.amepre.2009.08.028.

Lim, S. S., T. Vos, A. D. Flaxman, et al. 2012. "A comparative risk assessment of burden of disease and injury attributable to 67 risk factors and risk factor clusters in 21 regions, 1990-2010: a systematic analysis for the Global Burden of Disease Study 2010." Lancet 380 (9859):2224-60. doi: 10.1016/S0140-6736(12)61766-8.

Munson, S. A., H. Cavusoglu, L. Frisch, et al. 2013. "Sociotechnical Challenges and Progress in Using Social Media for Health." J Med Internet Res 15 (10):e226. doi: 10.2196/jmir.2792.

Nahm, E. S., J. Warren, S. Zhu, et al. 2012. "Nurses' self-care behaviors related to weight and stress." Nurs Outlook 60 (5):e23-31. doi: 10.1016/j.outlook.2012.04.005.

Ng, J. Y. Y., N. Ntoumanis, C. Thøgersen-Ntoumani, et al. 2012. "Self-Determination Theory Applied to Health Contexts: A Meta-Analysis." Perspectives on Psychological Science 7 (4):325-340. doi: 10.1177/1745691612447309.

Novak, R. D., and S. E. Auvil-Novak. 1996. "Focus Group Evaluation of Night Nurse Shiftwork Difficulties and Coping Strategies." Chronobiology International 13 (6):457-463. doi: 10.3109/07420529609020916.

Person, M., and J. Mårtensson. 2006. "Situations influencing habits in diet and exercise among nurses working night shift." Journal of Nursing Management 14:414-423.

Quintiliani, L., J. Sattelmair, and G. Sorensen. 2007. The workplace as a setting for interventions to improve diet and promote physical activity. Word Health Organization, Geneva. 
Ryan, R. M., and E. L. Deci. 2000. "Self-determination theory and the facilitation of intrinsic motivation, social development, and well-being." The American Psychologist 55 (1):68-78. doi: 10.1037/0003-066X.55.1.68.

Ryan, R. M., H. Patrick, E. L. Deci, et al. 2008. "Facilitating health behaviour change and its maintenance: Interventions based on self-determination theory." The European Health Psychologist 10 (1):2-5.

Schluter, P., C. Turner, A. D. Huntington, et al. 2011. "Work/life balance and health: the Nurses and Midwives e-cohort Study." International Nursing Review 1 (1466-7657 )$: 28-36$.

Stafford, M., S. Cummins, A. Ellaway, et al. 2007. "Pathways to obesity: Identifying local, modifiable determinants of physical activity and diet." Social Science \& Medicine 65 (9):1882-1897. doi: http://dx.doi.org/10.1016/j.socscimed.2007.05.042.

Sumaira, M., B. Holly, and B. Mark. 2011. "How healthy are our nurses? New and registered nurses compared." British Journal of Nursing 8:489-496.

Teixeira, P. J., E. V. Carraça, D. Markland, et al. 2012. "Exercise, physical activity, and selfdetermination theory: A systematic review." International Journal of Behavioral Nutrition and Physical Activity 9 (78). doi: 10.1186/1479-5868-9-78.

Torquati, L., M. Leveritt, T. Pavey, et al. 2015. "Promoting diet and physical activity in nurses: a systematic review." American Journal of Health Promotion (In press) (In press).

Turner-McGrievy, G. M., M. W. Beets, J. B. Moore, et al. 2013. "Comparison of traditional versus mobile app self-monitoring of physical activity and dietary intake among overweight adults participating in an mHealth weight loss program." Journal of the American Medical Informatics Association 20 (3):513-518. doi: 10.1136/amiajnl2012-001510.

Verweij, L. M., J. Coffeng, W. van Mechelen, et al. 2011. "Meta-analyses of workplace physical activity and dietary behaviour interventions on weight outcomes." Obes Rev 12 (6):406-29. doi: 10.1111/j.1467-789X.2010.00765.x.

Wendel-Vos, W., M. Droomers, S. Kremers, et al. 2007. "Potential environmental determinants of physical activity in adults: a systematic review." Obesity Reviews 8 (5):425-440. doi: 10.1111/j.1467-789X.2007.00370.x.

WHO. 2010. "WHO Healthy Workplace Framework and Model: Background and Supporting Literature and Practices." 
Williams, G. C., D. S. Minicucci, R. W. Kouides, et al. 2002. "Self-determination, smoking, diet and health." Health Education Research 17 (5):512-521. doi: 10.1093/her/17.5.512.

Wong, H., M. C. Wong, S. Y. Wong, et al. 2010. "The association between shift duty and abnormal eating behavior among nurses working in a major hospital: a cross-sectional study." Int J Nurs Stud 47 (8):1021-7. doi: 10.1016/j.ijnurstu.2010.01.001.

Zapka, J. M., S. C. Lemon, R. P. Magner, et al. 2009. "Lifestyle behaviours and weight among hospital-based nurses." J Nurs Manag 17 (7):853-60. doi: 10.1111/j.13652834.2008.00923.x.

Zhao, I., F. Bogossian, S. Song, et al. 2011. "The association between shift work and unhealthy weight: a cross-sectional analysis from the Nurses and Midwives'e-cohort Study." Journal of occupational and environmental medicine 53 (2):153-158. 


\section{Tables}

Table 1. Question guide

\begin{tabular}{ll}
\hline \multicolumn{1}{c}{ Type of question } & \multicolumn{1}{c}{ Question } \\
\hline Opening & $\begin{array}{l}\text { Tell us a bit about yourselves, your names and what made you } \\
\text { chose nursing as a profession }\end{array}$ \\
\hline Transition & What do you think defines a healthy lifestyle? \\
\hline \multirow{3}{*}{ Key } & What does physical activity mean to you? \\
& What motivates you to engage in physical activity? \\
& What is a healthy diet to you? \\
& Did your lifestyle change after you started to work as a nurse? \\
& Which strategies do you thing may help nurses improve their \\
Ending & diet and physical activity? \\
\hline
\end{tabular}


Table 2. Participants' characteristics

\begin{tabular}{lc}
\hline Gender & $82 \%$ female \\
Mean age (range) (years) & $39.5(25-59)$ \\
Education (n) & 2 \\
Graduate diploma & 10 \\
Bachelor of Nursing & 2 \\
Master of Nursing & $14.3(1-35)$ \\
Average $\quad$ working experience $\quad-$ years \\
$\begin{array}{l}\text { (range) } \\
\text { Role } \quad \text { Registered Nurse }\end{array}$ \\
Clinical Nurse \\
Shift worked \\
Day shift only
\end{tabular}






Figure 1. Interplay of diet and physical activity determinants and SDT 